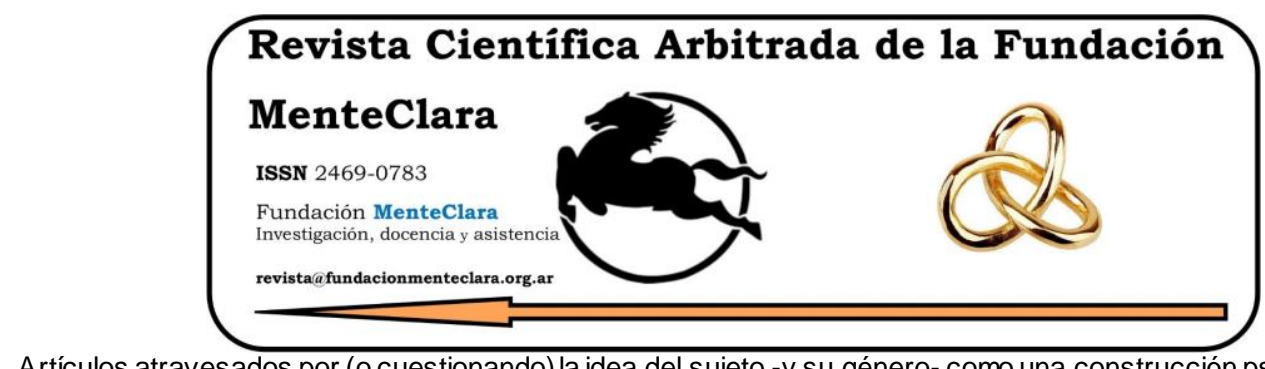

Artículos atravesados por (o cuestionando) la idea del sujeto -y su género- como una construcción psicobiológica de la cultura. Articles driven by (or questioning) the idea of the subject -and their gender- as a cultural psychobiological construction.

Vol. 6 (2021), enero-diciembre ISSN 2469-0783

https: / / datahub.io/ dataset/2021-6-e238

\title{
LA IMPORTANCIA DE LA PRESENCIA DE LA FAMILIA EN LAS INSTITUCIONES QUE ALBERGAN A LAS PERSONAS MAYORES
}

\author{
THE IMPORTANCE OF THE PRESENCE OF THE FAMILY IN THE INSTITUTIONS \\ THAT HOUSE THE ELDERLY
}

Silvana Baró ${ }^{1}$, Pablo Di Croce ${ }^{2}$, Romina Gómez González ${ }^{3}$, Zaida Nadal ${ }^{4}$

Cómo citar este artículo / Citation: Baró, S., Di Croce, P., Gómez González, R. y Nadal, Z. (2021). La importancia de la pre sencia de la familia en las instituciones que albergan a las personas mayores. Revista Cientifica Arbitrada de la Fundación MenteClara, Vol. 6 (238). DOI: https://doi.org/10.32351/rca.v6.238

Copyright: (C) 2021 RCAFMC. Este artículo de acceso abierto es distribuido bajo los términos de la licencia Creative Commons Attribution 4.0 International License (CC BY 4.0). Recibido: 16/07/2021. Aceptado: 20/07/2021 Publicación online: 21/07/2021

Conflicto de intereses: Ninguno que declarar.

\section{Resumen}

En situaciones como necesidades de vivienda o de cuidados respecto de su salud, el vínculo con la familia es fundamental para la salud integral y el bienestar psicológico. El objetivo de este artículo es visualizar la importancia del vínculo de la familia con el adulto mayor institucionalizado desde los trabajadores de los hogares y residencias. A partir de entrevistas a equipos interdisciplinarios que se desempeñan en las instituciones de adultos mayores se concluye que la falta de la familia en las

\footnotetext{
${ }^{1}$ Universidad de Flores. Facultad de Psicología y Ciencias Sociales, Argentina. silvana_baro@yahoo.com.ar

2 Universidad de Flores. Facultad de Psicología y Ciencias Sociales, Argentina. padicroce77@gmail.com

${ }^{3}$ Universidad de Flores. Facultad de Psicología y Ciencias Sociales, Argentina. rominagg86@gmail.com

4 Universidad de Flores. Facultad de Psicología y Ciencias Sociales, Argentina. zaidanadal@gmail.com
} 
instituciones, especialmente públicas, afecta la salud de los adultos mayores ya que se pierden las redes de apoyo social tan importantes en esta etapa de la vida.

\begin{abstract}
In situations such as housing or health care needs, older adults enter long-stay institutions and maintaining the bond with the family is essential for comprehensive health and psychological well-being. The objective of this article is to visualize the importance of the bond of the family with the institutionalized elderly from the workers of the homes and residences. Based on interviews with interdisciplinary teams that work in institutions for the elderly, it is concluded that the lack of the family in institutions, especially public ones, affects the health of the elderly since the social support networks so important in this stage of life.
\end{abstract}

Palabras Claves: Residencias de adultos mayores; Familia; Redes de apoyo social Keyw ords: Residences for the elderly; Family; Social support networks 


\section{Introducción}

En el marco de la investigación "El trabajo interdisciplinario en hogares y residencias de adultos mayores" de la Ciudad Autónoma de Buenos Aires (Baró et al., 2021), se indagó acerca de la articulación del equipo interdisciplinario con las familias y los lazos que mantenían con los residentes en instituciones públicas y privadas. Cabe agregar, que el objetivo de este estudio fue describir y analizar el trabajo del equipo interdisciplinario en instituciones de adultos mayores de CABA.

Es de considerar que las personas mayores conforman una población en crecimiento tanto en Argentina como en el mundo. El avance de las ciencias ha procurado un mayor conocimiento sobre las distintas enfermedades y ha ayudado a extender los años de vida. Arlegui (2009) citado en Lalive D'Epinay et al. (2005) refiere que en la segunda etapa del envejecimiento, que sitúa alrededor de los 80 años, puede producirse un estado de fragilidad que necesitaria recurrir a ciertos recursos sanitarios, sociales y económicos en donde la familia ocupa un factor importante. La autora agrega que aquéllos que viven solos y con más edad se encuentran en situación de mayor riesgo.

Por esta razón, en situaciones en donde se requiere una vivienda, una atención integral de manera continua llevada a cabo por un personal capacitado, el adulto mayor entra en una residencia, ya sea para cubrir necesidades de vivienda o de cuidados respecto de su salud.

Una residencia puede definirse como un "centro gerontológico abierto de desarrollo personal y atención sociosanitaria multiprofesional en el que viven temporal o permanentemente personas mayores con algún grado de dependencia." (Rodriguez, 2004 en Croas y Fernandez Colmeneros, 2013). 
Como institución abierta, una residencia debe garantizar una interacción bidireccional hacia fuera de la residencia y hacia adentro. Es decir, tiene que erigirse como Centro Gerontológico Abierto, haciendo posible la entrada y la participación en las actividades a familiares, amigos, allegados y a otras personas o grupos de la comunidad.

El rol de la familia en el entorno del adulto mayor está vinculado al concepto de redes de apoyo social. Existen redes de apoyo formal, brindadas por instituciones públicas y privadas, y redes informales, compuestas por familiares, amigos, vecinos, etc. Así, este concepto alude a todas las relaciones interpersonales, familiares y no familiares, capaces de brindar algún tipo de apoyo, ya sea emocional, práctico, económico, de compañía, guía o consejo. (Villanueva y Fernandez Salvador, 2010) (Arias, s/f).

En relación a la institucionalización de los adultos mayores y su vínculo con las familias, Oyola Galvan y Blanquiet Ulloa (2018) destacan que la decisión de institucionalizar al familiar mayor se debe mayormente al desmejoramiento de su salud y a la carga que representaba para ellos, asimismo, manifiestan que, con el paso del tiempo de permanecer en la residencia, el vínculo afectivo se va desgastando lo que puede desencadenar el rompimiento de los lazos.

La institución condiciona la frecuencia, intensidad y tipo de interacción que se establece con las familias. Dependerá del grado de flexibilidad o rigidez de sus normativas, a la posibilidad de generar áreas de participación entre familia e institución. Pueden suscitarse conflictos entre familia y profesionales respecto a los espacios de participación y decisión sobre los adultos mayores, que pueden generar demandas que se traducen en fuentes de estrés para los profesionales. Por ejemplo, 
familias que intervienen en las tareas del profesional, criticas injustificadas, aumento de demandas, exigencias inapropiadas. La implicación familiar está condicionada por cada familiay en especial, por el perfil de cuidador que tengan. Esto es útil tener en cuenta ya que es preferible contar con el apoyo familiar como complemento de cuidados y apoyo social (Gonzalez Antolin y Dominguez Llorente, 2000).

\section{Materiales y Método}

El presente artículo se elaboró en base a uno de los resultados de una investigación más amplia llevada a cabo en instituciones privadas y públicas que albergan a personas mayores en la Ciudad de Buenos Aires, más precisamente, dos instituciones públicas y una privada. Para este estudio se utilizó una metodología cualitativa con un diseño descriptivo y transversal y una estrategia de estudio de caso. Para ello se tomaron 31 entrevistas semidirigidas y en profundidad a los distintos trabajadores que se desempeñan en las instituciones, el criterio de inclusión para participar como sujetos de la investigación fue trabajar en la institución y estar en contacto con los adultos mayores. Asimismo, se realizaron observaciones de reuniones de equipo interdisciplinario y de asamblea con los residentes. Previamente se contactó a los directores de todas las instituciones -hogares y residencias- quienes fueron presentando a sus equipos, a cada uno de los entrevistados se les presentó previamente el consentimiento informado. El análisis de los datos se realizó teniendo en cuenta las categorias, las que inspiraron este artículo, fueron dos de ellas: articulación de las familias y el equipo interdisciplinario y vínculo del adulto mayor y su familia 


\section{Resultados}

La investigación, cuyo objetivo principal era describir y analizar el trabajo interdisciplinario en hogares y residencias de adultos mayores, consideró entre sus categorias de análisis a la vinculación de las familias en relación con la salud integral del adulto mayor y a la articulación con las familias respecto del trabajo interdisciplinario.

Respecto del vínculo familiar con el residente, se encontró que algunas veces no existe o es poco frecuente, especialmente en los hogares de gestión pública. Los trabajadores de las residencias y hogares manifestaron la importancia de mantener el contacto con la familia para el bienestar psicológico de los residentes y, especialmente en el sostenimiento de los vínculos afectivos. Sin embargo, ese contacto se dificulta cuando no hay familia o su presencia es escasa o nula. Así lo expresaba la trabajadora social de una de las residencias públicas: “...de los que estánentrando vienen de paradores, de comedores, de iglesias, no hay familias, están desarticuladas, estamos viviendo en una sociedad..."

Asimismo, el personal directivo de otra de las residencias públicas manifestó: "Hay una especie de agujero negro. No sé si a todas las residencias les pasa, como para que en unas entrevistas que estuve haciendo de experiencia de otras personas, no ve al familiar". Más adelante en la entrevista, agregó que los familiares dicen: "no lo puedo, no lo reconozco, me da tanta pena verlo asî" y les dice: "y vos no sabés la pena que tiene que no vengas".

Aunque, se observó que en la residencia privada había más presencia familiar en actividades y en los espacios comunes. En una observación de una actividad llevada a cabo por la psicóloga de la institución, familiares que visitaban a los residentes participaron de la reunión donde 
se trataban diversos temas de interés coordinados por la profesional que favorecía la participación de residentes y familiares.

Respecto del trabajo de articulación del equipo interdisciplinario con las familias en una de los hogares públicos, la directora señaló:

Pasa en todos lados, igual estamos convocando y convocando, mi propuesta es llamar al familiar y dar un breve informe de cómo está el familiar -papá, abuelo- si quiere llamarla por teléfono "hola llamo por tal cosa" preguntarle si quiere decirle algo. La cara que pone la gente cuando se le dice "recién hablé con tu hijo" es fundamental. Muchas veces piensan que debe estar enojado o algo le molestó por eso no viene

Si bien, en la residencia privada se observó más presencia y articulación con las actividades propuestas por los profesionales, se observó en numerosas entrevistas, tanto en privada como en públicas, la necesidad de contar con más presencia de las familias, como algo a construir desde el personal de los hogares y residencias para con las familias de los residentes. En relación a la articulación con las familias, la coordinadora de laborterapia de una de las instituciones públicas manifestó:

...hablamos del tema que siempre se intenta involucrar a la familia pero en su momento yo planteé armar encuentros, un encuentro de juegos, un sábado para que la familia pueda venir y empezar a llamar y ver, además de la familia, amigos, alguien que tenga relación con el residente y bueno en su momento "y no porque no van a poder"...

Así también, la profesional señala:

...es verdad hay gente que no la tiene, hay gente que la tiene pero no tiene relación, pero quizás hay gente que no tiene a su familia pero tienen contención, amigos, fue armando...acá hay casos, entonces quizás sería 
interesante para intentar incluir a la familia desde ese lugar y que se sientan más parte...

Cabe agregar que una integrante del equipo de trabajo social de una de las instituciones públicas, señaló:

...hay familias que son presentes, otras que no, pero digo, justamente una particularidad que observé es que con las familias que están más presentes los residentes no requieren de muchas intervenciones, a ver.... uno va digo y realiza sus funciones, pero digo no hay una intervención de urgencia...

\section{Discusión}

Contar con la red de apoyo social, en este caso la familia y los amigos, para Sluzki (1998), significa tener apoyo emocional que el autor señala como una de las funciones de la red, es decir, que la persona pueda interactuar con otros y que eso se traduzca en estímulo y apoyo.

Asimismo, la investigación de Silva Imbachi y Meneces Osorio (2018) señala que la institucionalización no sustituye la presencia y el afecto que proporciona la familia y su ausencia puede llevar al adulto mayor a estados de ansiedad y depresión.

En relación a la ausencia Oyola Galvan y Blanquiet Ulloa (2018), sostiene que con el devenir del tiempo transcurrido de la internación se genera un desgaste en el vínculo afectivo, que deja en evidencia una profunda disociación en las relaciones.

En concordancia con lo señalado, Arias (2009), manifiesta que se ha demostrado en diversas investigaciones la importancia que reviste la 
interacción con la familia y la comunidad del adulto mayor para su salud integral y su bienestar psicológico.

\section{Conclusiones}

La presencia de la familia en interacción con las personas mayores institucionalizadas determina un factor importante para su salud integral. Tanto los profesionales de la salud como los trabajadores que están en contacto diario con los residentes reconocen la importancia del sostenimiento del vínculo familiar luego de la institucionalización. A su vez, advierten que presenta dificultades y obstáculos el sostener la participación y la relación de la familia con el adulto mayor. Asimismo, se observó la búsqueda de distintas alternativas desde los trabajadores de las residencias, sin embargo, requiere de familias dispuestas a sostener el vínculo.

Se recomienda seguir investigando con familias como sujetos de investigación y no desde la perspectiva de los equipos interdisciplinarios que se desempeñan en las instituciones, a través de entrevistas en profundidad que ayuden a comprender este fenómeno.

La entrada a un hogaro una residencia implica un pasaje hacia nuevas formas de vivir, y en ese pasaje, muchas veces, la familia no acompaña y es cuando más se necesita. 


\section{Referencias}

Arlegui, M. (2009). Situación de los adultos mayores en la Argentina Activos y capacidad de respue sta frente a la vejez en contextos vulnerables Congreso Internacional de la Asociación Latinoamiericana de Sociología. Disponible en: https:/ / www.gerontologia.org/portal/archivosUpload/Situacion-adultos-mayoresArgentina.pdf

Arias, C. J. (s/f). El apoyo social en la vejez. En: Contenidos Gerontológicos para la formación de cuidadores domiciliarios. Ministerio de Desarrollo Social.

Arias, C. (2009). La red de apoyo social en la vejez. Aportes para su evaluación. Revista de Psicologia da IMED, vol.1, n.1, p. 147-158

Baró, S., Godoy, M., Sorbara, S., Stambuli, S., Petrongolo, M., Di Croce, P., Gómez González, R., Mielnik, F., Nadal, Z., Passarelli, M., Rudoni, N., Seminaroti, F. (2021). El trabajo interdisciplinario en hogares y residencias de la Ciudad Autónoma de Buenos Aires. [No publicado]. Universidad de Flores

Croas, R. y Fernandez Colmeneros, L. (2013). Modelos Comunitarios e Instituciones de Gestión Gerontológica. En: Especialización en Gerontología Comunitaria e Institucional. Ministerio de Desarrollo Social. Presidencia de la Nación - Universidad de Mar del Plata. Disponible en: https://www.desarrollosocial.gob.ar/wp-content/uploads/2015/05/Gerontolog--aComunitaria-Modulo-51.pdf

González Antolín, E., Domínguez Llorente, M. L. (2000). Factores que inciden en la actuación profe sional con personas mayores. Papeles de1 Psicólogo, núm. 76, pp. 9-12.

Oyola Galvan, Y. y Blanquiet Ulloa, V. (2018). Relaciones y vínculos afectivos entre las fa milias y los ancianos institucionalizados del Hogar Geriátrico Sagrado Corazón de Jesús en vos confio (Ciénaga-Magdalena). [Tesis de grado]. Universidad Cooperativa de Colombia. Disponible en: https:/ / re pository.ucc.edu.co/bitstream/20.500.12494/5903/1/Relaciones\%20y\%20vinculo s\%20afectivos.pdf

Silva Imbachi, J. y Meneces Osorio, M. (2018). Influencia de los factores familiares en la calida de vida de los 8 adultos mayores institucionalizados en la casa Hogar Villa Carolina de Timbio Cauca. [Tesis de grado]. Fundación Universitaria de Popeyán. Disponible en: http:/ / unividafup.edu.co/repositorio/files/original/396ca5678393ef96ae 1f1fe 5c7621797.pdf

Sluzki, C. (1998). La red social: proposiciones generales. En: La red social: Frontera de la práctica sistémica. Barcelona. Gedisa

Villanueva, A., \& Fernandez Salvador, C. (2010). Las personas mayores y su entorno social, económico y cultural. En: M. Roqué (comp.) Manual de cuidados domiciliarios: Nuevos paradigmas en politicas sociales. Nuevos escenarios gerontológicos. Disponible en: https://fiapam.org/wp-content/uploads/2012/10/cui da dores-cuader-1-we b.pdf 